\title{
Cambios morfométricos en el nervio oculomotor del perro durante el desarrollo posnatal
}

\author{
Morphometric changes in the dog oculomotor nerve with postnatal growth
}

\author{
J Vivo*, F Miró, AM Galisteo, E Agüera, C Plaza, A Díz
}

\begin{abstract}
The aim of the study was to investigate aged-related changes in morphometric features both in the myelinated and unmyelinated fibers of the oculomotor nerve in dogs. According to their age (1,3,5, 7 weeks and 4 years) twenty Beagles were classified into five groups (A, B, C, D, and E, respectively). The right intracranial portions of the nerves were processed and analysed by light and electron microscopy. In every nerve, cross-sectional area was calculated. In the myelinated fibers the number, diameter and cross sectional area were calculated, as well as the cross-sectional area and diameter of their axons, and myelin sheath thickness. In unmyelinated fibers the number, cross sectional area and diameter were calculated. Graphical representations for every variable were carried out by mean of frequency histograms. The cross sectional area of the nerve increased in size 4.5 times from group A to group E. From group A to group E the number of myelinated fibres increased significantly 1.5 times and its diameter 2.3 times. The number of unmyelinated fibres decreased in $20 \%$ and its diameter $40 \%$ from group A to group E. According to the results obtained the oculomotor nerve of the dog has similar structural features than that of other mammal species, and this nerve modifies its morphometry with growth. The results obtained could improve the understanding of those possible pathological conditions that involve morphological alterations of the nerve fibers.

Key words: oculomotor nerve, dog, development, morphometry.
\end{abstract}

\begin{abstract}
RESUMEN. El objetivo fue investigar los cambios relacionados con la edad en las características morfométricas de las fibras mielínicas y amielínicas del nervio oculomotor en el perro. Veinte perros Beagles se distribuyeron, según edad (1, 3, 5, 7 semanas y 4 años), en cinco grupos (A, B, C, D, y E, respectivamente). Se extrajeron los nervios en su porción intracraneal derecha, siendo procesados y analizados por microscopia óptica y electrónica. Se calculó el área de la sección transversal de cada nervio. En las fibras mielínicas de cada uno de los nervios se determinó su número, diámetro y área de sección transversal, así como el área de sección transversal y diámetro de sus axones y grosor de la vaina de mielina. Por su parte, en las fibras amielínicas se calculó el número, área de su sección transversal y diámetro. Se realizaron histogramas de frecuencia para todas las variables. El área de la sección transversal del nervio aumentó en 4,5 veces el tamaño del grupo A al grupo E. Del grupo A al grupo E el número de fibras mielínicas aumentó significativamente 1,5 veces y 2,3 veces su diámetro, mientras que el número de fibras amielínicas disminuyó $20 \%$ y su diámetro $40 \%$ del grupo A al grupo E. El nervio oculomotor del perro posee características estructurales similares en otras especies de mamíferos, y modifica su morfometría con el desarrollo. Los resultados obtenidos podrían mejorar la comprensión de aquellos trastornos patológicos que cursen con alteraciones de las características morfológicas de las fibras.

Palabras clave: nervio oculomotor, perro, desarrollo, morfometría.
\end{abstract}

\section{INTRODUCCIÓN}

Los nervios oculomotores (abducente, troclear y oculomotor), responsables de los movimientos de los ojos, se componen de fibras mielínicas y amielínicas que presentan diferentes características morfológicas y velocidad de conducción (Manni y col 1987, 1989). Las fibras nerviosas del nervio oculomotor (n. oculomotorius) se originan en el nucleus motorius $n$. oculomotorii y en el nuclei parasympathici $n$. oculomotorii, ambos ubicados en el tronco del encéfalo (Brodal 1981). El nervio oculomotor sale del tronco del encéfalo por la superficie ventromedial del mesencéfalo, justo medial al pedúnculo cerebral, alcanzando inmediatamente la órbita por medio de la cisura orbitaria y se divide en los ramos dorsal y ventral (König y col 2014).

Aceptado: 15.10.2015.

Departamento de Anatomía y Anatomía Patológica Comparadas, Campus Rabanales, Universidad de Córdoba, Córdoba, España.

*Autor de correspondencia: J Vivo; an1viroj@uco.es
El nervio oculomotor inerva cinco músculos extraoculares (recto ventral, recto dorsal, recto medial, oblicuo ventral y elevador del párpado superior), siendo estos músculos los más activos en los movimientos del globo ocular. Mientras que las fibras nerviosas con grandes axones y gruesa capa de mielina son generalmente responsables de la conducción rápida de los impulsos y de los movimientos rápidos de los ojos, otras fibras, de medio y pequeño tamaño, son responsables de la contracción sostenida, como se requiere en la fijación ocular (Bardosi y col 1990).

Los estudios realizados sobre el nervio oculomotor se han centrado en diferentes especies. Bortolami y col (1977) en ovejas, perros y gatos, Manni y col (1978, 1987, 1989) en el gato y las ovejas, Porter y Spencer (1982) y Hayakawa y col (2000) en el gato, y Lanzino y col (1993) en el ser humano han analizado los aspectos funcionales de los nervios. Otros autores como Batini y col (1979) en el gato, Fraher (1989 a,b) en la rata, Berardinelli y col (2000) en ovejas y Kawamura y col (2000) y Sharma y col (2009) en humanos han investigado los aspectos morfológicos de sus fibras. Sharma y col (2009) demostraron que los 
cambios morfológicos con el crecimiento pueden provocar alteraciones en la velocidad de conducción de los impulsos en el nervio oculomotor y Mayhew y Sharma $\left(1984^{\mathrm{a}, \mathrm{b}}\right)$ y Kawamura y col (2000) sugieren una reducción numérica de fibras mielínicas y amielínicas. En los perros, el estudio de las características de los nervios oculomotores ha recibido poca atención de los investigadores. Bortolami y col (1977) examinaron el nervio oculomotor, Vivo y col (2004) analizaron algunas variables de las fibras mielínicas y amielinizadas en el nervio troclear de perros adultos y Vivo y col (2006) analizaron el de los tres nervios oculomotores. A saber, el único estudio que analiza las características de la morfología de los tipos de fibras durante el crecimiento se realizó por Vivo y col (2013) en el nervio troclear.

Las fibras nerviosas mielínicas se caracterizan por su vaina de mielina y al microscopio óptico muestran diferencias considerables en su tamaño. Por su parte, las fibras amielínicas carecen de vaina de mielina, tienen morfología ovalada o redondeada, con tamaños variados y se identifican con microscopia electrónica distribuidas entre las fibras mielínicas.

Con este estudio se pretende contribuir al conocimiento de algunas características de las fibras mielínicas y amielínicas del nervio oculomotor en el perro Beagle durante el crecimiento que puedan servir de referencia en el diagnóstico de aquellas patologías que cursan con alteraciones de estas características morfológicas.

\section{MATERIAL Y MÉTODOS}

Se utilizaron veinte Beagles sanos divididos en cinco grupos (A, 7 días; B, 21 días; C, 35 días; D, 49 días y E, 4 años de edad) de cuatro perros cada uno. Los perros se obtuvieron del Servicio Centralizado de Experimentación Animal de la Universidad de Córdoba, donde fueron criados en jaulas al aire libre con comida y agua a libre disposición. Fueron sacrificados humanitariamente, utilizando isoflurano como anestesia, seguido de un barbitúrico intravenoso según la legislación vigente en experimentación animal (Directiva 2010/63 / UE, y Real Decreto 1201/2005, de 10 de octubre). Este estudio fue aprobado por el Comité de Bioética de Experimentación Animal de la Universidad de Córdoba ( 2 de febrero $2012 n^{\circ} 712$ ). Tras el sacrificio, se eliminó la bóveda del cráneo a fin de obtener la porción intracraneal derecha del nervio oculomotor.

Los nervios se fijaron en una solución al $2 \%$ de glutaraldehído en un buffer fosfato $0,1 \mathrm{M}(\mathrm{pH} 7,4)$ durante la noche a $4^{\circ} \mathrm{C}$ y luego fijados durante 30 minutos en una solución de tetróxido de osmio al $1 \%$ preparada en un buffer fosfato $0,1 \mathrm{M}$ (pH 7,4). Después de la deshidratación en gradientes de etanol fueron incluidos en araldita y cortados con un ultramicrotomo LKB (Leica Reichert, Viena. Austria) en secciones transversales semifinas y ultrafinas. Las secciones semifinas se tiñeron con azul de toluidina y fueron estudiadas con microscopio óptico, las secciones ultrafinas fueron doblemente teñidas con acetato de uranilo y citrato de plomo. Las secciones ultrafinas fueron examinadas y fotografiadas en un microscopio electrónico de transmisión Philips CM10 (Philips, Mahwah, NJ, EE.UU.).

Las secciones semifinas de los nervios enteros fueron digitalizadas en sucesivas imágenes a 40x, con las que se reconstruyó completamente la sección del nervio. Las secciones ultrafinas se analizaron a $10.000 x$ y fueron fotografiadas a $25.000 x$ y seguidamente almacenadas. Sobre la reconstrucción completa del nervio se contó el número de todas las fibras mielínicas y se midió el área del nervio utilizando el software Scion Image (1998 Scion Corporation. Frederick. MD. EE.UU.). Además se seleccionaron imágenes equivalentes a una superficie del $10 \%$ del nervio completo, en las que se midió el área de sección transversal, el diámetro máximo y mínimo de las fibras mielínicas, y de sus axones correspondientes. De igual modo se procedió sobre las secciones transversales ultrafinas, en las que se contaron el número de fibras amielínicas y se calculó el área de su sección transversal, así como sus diámetros máximo y mínimo.

Los datos resultantes se extrapolaron a todo el nervio con una simple regla aritmética. Los datos fueron analizados utilizando el programa SPSS 15.0 ® para el software de Windows ${ }^{\circledR}$ para obtener, a partir de cada nervio, para las fibras mielínicas y amielínicas, el porcentaje de cada tipo de fibra, el diámetro medio fibrilar, la densidad (número de fibras por $\mathrm{mm}^{2}$ ), área media, área total (suma del área de todas las fibras) y el área relativa (expresada en porcentaje del área del nervio). Por otra parte, en las fibras mielínicas se calcularon el área media del axón y su diámetro medio, grosor de la vaina de mielina ([diámetro fibrilar menos diámetro del axón] / 2) y la razón diámetro del axón / diámetro fibrilar. Con el fin de estudiar la frecuencia de los datos todas las variables se representaron gráficamente mediante histogramas. Para cada nervio y para las variables que procedieran se obtuvo la desviación estándar (intra std). En cada grupo de edad estudiado se calculó la media global (obtenida a partir de la media de cada nervio) y su correspondiente desviación estándar (std inter) y la media de la desviación estándar intra. Para determinar las diferencias estadísticas entre los grupos de edad se llevó a cabo un ANOVA y una prueba posthoc DMS.

\section{RESULTADOS}

\section{FIBRAS MIELIINICAS}

Las fibras nerviosas mielínicas se identificaron gracias a las características de su vaina de mielina, al microscopio óptico, y como se muestra en la figura 1 se aprecian diferencias considerables en su tamaño. El diámetro de la fibra (figura 3) y el espesor de la vaina de mielina en el grupo E presentaban una distribución bimodal en los histogramas. Por su parte, las demás variables de este grupo presentaron una distribución unimodal. Respecto de 
todas las variables en los otros grupos, su distribución fue unimodal. Las fibras, independientemente de su tamaño, se encuentran repartidas al azar en toda la sección del nervio.

Los resultados obtenidos mostraron que los nervios aumentaron su área media con la edad, siendo para el grupo A significativamente menor que para los grupos $\mathrm{C}, \mathrm{D}$ y $\mathrm{E}(\mathrm{P} \leq 0,05)$. Es interesante señalar que el valor de esta variable fue 4,25 veces mayor en el grupo E que en el grupo A (cuadro 1).

En los grupos B, C y E el número de fibras mielínicas es significativamente mayor que en el grupo A.

Los nervios de los perros de 7 días de edad (grupo A) tenían valores medios significativamente menores para el espesor de la vaina de mielina, el diámetro del axón, el área del axón, el área de la fibra y el diámetro de la fibra, que los nervios de los perros pertenecientes a los grupos, B, C, D y E. En consecuencia, el incremento en el valor de estas variables está relacionado con la edad (cuadro 1, figura 2).

El área relativa de las fibras respecto de la sección del nervio no muestra diferencias significativas entre los grupos, pero se observó un aumento con la edad (cuadro 1).

Los histogramas de frecuencias mostraron una diferente distribución para el diámetro de las fibras en los distintos grupos de edad. El mayor porcentaje de fibras en el grupo A $(25 \%)$ estaban en el intervalo entre 4 y $4,5 \mu \mathrm{m}$, en el grupo B $(17,4 \%)$ entre 5 y $5,5 \mu \mathrm{m}$, en el grupo C $(13,6 \%)$ entre 6,5 y $7 \mu \mathrm{m}$, en el grupo $\mathrm{D}(8,5 \%)$ entre 8,5 y $9 \mu \mathrm{m}$. El grupo E tenía el 5,7\% tanto entre 5 y 5,5 y como entre 6 y $6,5 \mu \mathrm{m}$. Las dos poblaciones de fibras (distribución

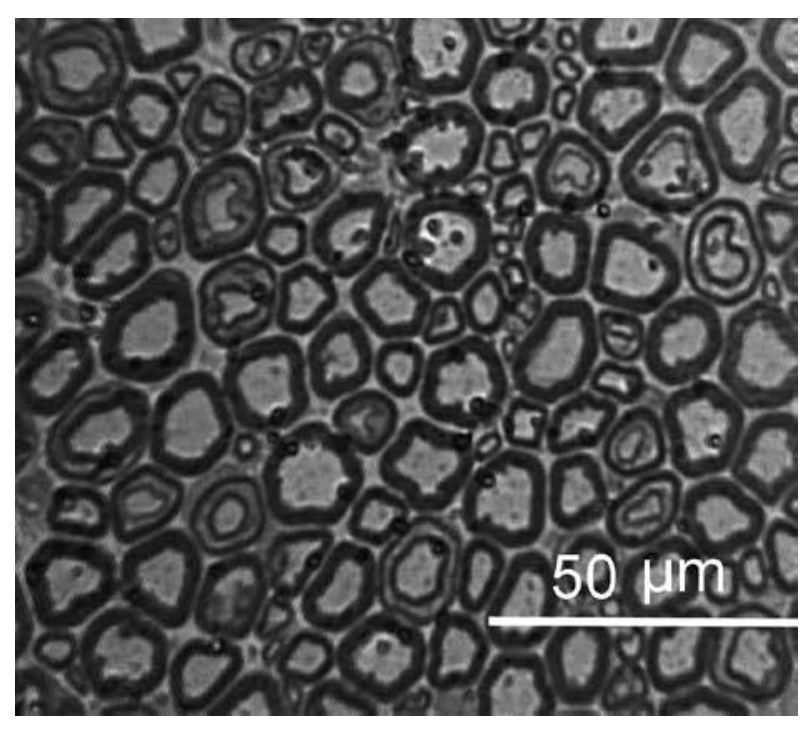

Figura 1. Micrografía óptica de un corte transversal (40x) del nervio oculomotor en el grupo E. Se pueden observar las diferencias en el tamaño de las fibras mielínicas.

Light micrograph of transverse section (40x) of the oculomotor nerve in group E. Differences in size of myelinated fibres can be observed.

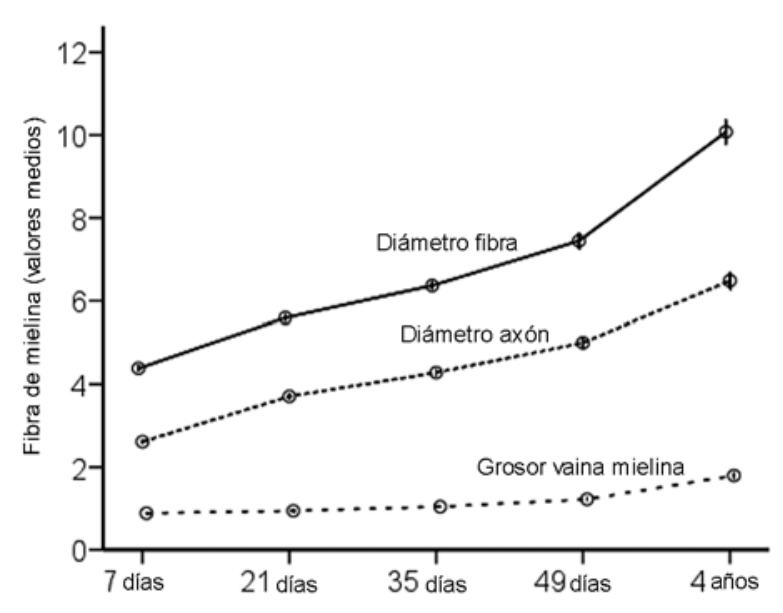

Figura 2. Evolución con la edad del diámetro fibrilar, diámetro del axón y del grosor de la vaina de mielina de las fibras mielínicas.

Evolution with age of the fibre diameter, axon diameter and myelin sheath thickness of myelinated fibres.

bimodal) del grupo E estaban separadas por el intervalo entre 10,5 y $11 \mu \mathrm{m}$.

Los rangos de espesor de la vaina de mielina fueron 0,43-1,7 $\mu \mathrm{m}$ para el grupo A, 0,34-1,79 $\mu \mathrm{m}$ para el grupo $\mathrm{B}, 0,35-2,60 \mu \mathrm{m}$ para el grupo $\mathrm{C}, 0,39-2,51 \mu \mathrm{m}$ para el grupo D y $0,48-4,82 \mu \mathrm{m}$ para el grupo E. Sus valores medios fueron $0,89 \mu \mathrm{m}, 0,94 \mu \mathrm{m}, 1,04 \mu \mathrm{m}, 1,22 \mu \mathrm{m}$ y $1,80 \mu \mathrm{m}$, respectivamente.

\section{FIBRAS AMIELÍNICAS}

Las fibras amielínicas, con morfología ovalada o redondeada, se identificaron mediante el uso de microscopia electrónica en todos los nervios de los cinco grupos de edad estudiados. Como se muestra en la figura 4 presentaban tamaños variados, y se encontraban distribuidas entre las fibras mielínicas.

Los histogramas de frecuencia de cada grupo (datos de cuatro muestras por grupo) evidenciaron que las variables área de la fibra y diámetro de la fibra presentaban una distribución unimodal. El mayor porcentaje de fibras en el grupo A (7,5\%) fue entre el intervalo 0,8-0,85 $\mu \mathrm{m}$, en el grupo B $(9,5 \%)$ entre 0,75 a $0,80 \mu \mathrm{m}$, en el grupo $\mathrm{C}$ $(13,6 \%)$ entre $03-0,35 \mu \mathrm{m}$, en el grupo D $(19,85 \%)$ entre 0,25 a $0,30 \mu \mathrm{m}$ y en el grupo $\mathrm{E}(17,3 \%)$ entre $0,2-0,25$ $\mu \mathrm{m}$ (figura 6).

Los grupos B, C, D y E presentan un número de fibras amielínicas significativamente menor que en el grupo A $(\mathrm{P} \leq 0,05)$. Los nervios de los perros de 7 días de vida presentaron para el diámetro de las fibras (figura 5), el área de las fibras y el área relativa de las fibras, valores medios significativamente más altos que los nervios de perros mayores. En consecuencia, la disminución en el valor de estas variables está relacionada con la edad (cuadro 1). 
VIVOY COL

Cuadro 1. Medias y desviaciones estándar de cada variable analizada de las fibras mielínicas y amielínicas de los cinco grupos estudiados.

Means and standard deviations of the myelinated and unmyelinated fibres of each analysed variable of the five groups studied.

\begin{tabular}{|c|c|c|c|c|c|c|c|c|}
\hline & M & Std inter & Std intra & $\mathrm{AM}$ & Std inter & Std intra & Total & Std inter \\
\hline \multicolumn{9}{|l|}{ GRUPO A (7días) } \\
\hline $\begin{array}{l}\text { Área media del nervio } \\
\left(\mu m^{2}\right)\end{array}$ & & & & & & & $311.446,99$ & $92.647,45^{\text {cde }}$ \\
\hline Número fibras & $6.702,67$ & $611,14^{\text {bce }}$ & & $1.894,79$ & 761,92 bcde & & & \\
\hline Porcentaje fibras & 77,96 & 8,34 & & 22,04 & 8,34 & & & \\
\hline Área fibras $\left(\mu \mathrm{m}^{2}\right)$ & 15,14 & $3,52^{\text {bcde }}$ & 5,69 & 0,61 & $0,21^{\text {bcde }}$ & 0,77 & & \\
\hline Área total fibras $\left(\mu \mathrm{m}^{2}\right)$ & $100.729,99$ & $19.497,28$ & & $1.047,12$ & 50,16 & & & \\
\hline Área relativa fibras (\%) & 33,11 & 4,28 & & 0,36 & 0,13 & & & \\
\hline Densidad fibras $\left(\mathrm{n}^{\circ} / \mathrm{mm}^{2}\right)$ & $23.103,65$ & $7.987,07$ & & $6.191,92$ & $1.953,84$ & & & \\
\hline Diámetro fibras $(\mu \mathrm{m})$ & 4,38 & 0,52 bcde & 0,81 & 0,78 & $0,12^{\text {bcde }}$ & 0,42 & & \\
\hline Área axón $\left(\mu \mathrm{m}^{2}\right)$ & 5,32 & 0,89 bcde & 2,27 & & & & & \\
\hline Diámetro axón $(\mu \mathrm{m})$ & 2,61 & $0,24^{\text {bcde }}$ & 0,55 & & & & & \\
\hline Grosor mielina $(\mu \mathrm{m})$ & 0,89 & 0,17 bcde & 0,20 & & & & & \\
\hline $\begin{array}{l}\text { Diam, axón/Diam, fibra } \\
\text { (um) }\end{array}$ & 0,60 & $0,04^{\text {bcde }}$ & 0,05 & & & & & \\
\hline
\end{tabular}
$(\mu \mathrm{m})$

GRUPO B (21 días)

Área media del nervio $\left(\mu \mathrm{m}^{2}\right)$

Número fibras

Porcentaje fibras

Área fibras $\left(\mu \mathrm{m}^{2}\right)$

Área total fibras $\left(\mu \mathrm{m}^{2}\right)$

Área relativa fibras (\%)

Densidad fibras $\left(\mathrm{n} / \mathrm{mm}^{2}\right)$

Diámetro fibras $(\mu \mathrm{m})$

Área axón $\left(\mu \mathrm{m}^{2}\right)$

Diámetro axón $(\mu \mathrm{m})$

Grosor mielina $(\mu \mathrm{m})$

Diam, axón/Diam, fibra $(\mu \mathrm{m})$

\section{GRUPO C (35 días)}

Área media del nervio $\left(\mu \mathrm{m}^{2}\right)$

Número fibras

Porcentaje fibras

Área fibras $\left(\mu \mathrm{m}^{2}\right)$

Área total fibras $\left(\mu \mathrm{m}^{2}\right)$

Área relativa fibras (\%)

Densidad fibras $\left(\mathrm{n}^{\mathrm{o}} / \mathrm{mm}^{2}\right)$

Diámetro fibras $(\mu \mathrm{m})$

Área axón $\left(\mu \mathrm{m}^{2}\right)$

Diámetro axón $(\mu \mathrm{m})$

Grosor mielina $(\mu \mathrm{m})$

Diam, axón/Diam, fibra $(\mu \mathrm{m})$

$\begin{array}{rcr}10.147,75 & 1.799,41^{\text {a }} & \\ 89,82 & 1,92 & \\ 24,74 & 5,62^{\text {acde }} & 9,98 \\ 47.006,19 & 46.747,51 & \\ 50,13 & 6,21 & \\ 20.919,40 & 4.601,82 & \\ 5,59 & 0,62^{\text {acde }} & 1,16 \\ 10,68 & 1,88^{\text {acde }} & 4,53 \\ 3,71 & 0,33^{\text {acde }} & 0,82 \\ 0,94 & 0,15^{\text {acde }} & 0,24 \\ 0,66 & 0,01^{\text {acde }} & 0,05\end{array}$

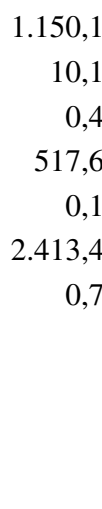

166,94 acde

$\begin{array}{rr}50,11 & 166,94 \\ 10,18 & 1,92 \\ 0,45 & 0,06\end{array}$

$493.659,0674.812,27^{\mathrm{e}}$

$517,69 \quad 121,64$

$0,11 \quad 0,04$

$2.413,45 \quad 596,82$

$0,70 \quad 0,02$ acde $\quad 0,32$

0,45

(

$626.679,5384.090,16^{\text {ae }}$

$10.761,20 \quad 2.372,22$

$86,51 \quad 5,89$

$32,25 \quad 3,23$ abde

13,63

$1.678,72$

590,19 abde

13,49

5,89

$345.641,01 \quad 74.119,15$

$54,83 \quad 6,13$

$17.083,63 \quad 2.099,54$

$6,37 \quad 0,36$ abde

$14,36 \quad 0,71$ abde

6,62

$4,28 \quad 0,16^{\text {abde }} \quad 1,05$

$1,04 \quad 0,13^{\text {abde }} \quad 0,29$

$0,67 \quad 0,02^{\text {abde }} \quad 0,06$

GRUPO D (49 días)

Área media del nervio $\left(\mu \mathrm{m}^{2}\right)$

Número fibras 
Continuación Cuadro 1.

\begin{tabular}{|c|c|c|c|c|c|c|c|c|}
\hline & M & Std inter & Std intra & AM & Std inter & Std intra & Total & Std inter \\
\hline Porcentaje fibras & 84,92 & 4,69 & & 15,08 & 4,69 & & & \\
\hline Área fibras $\left(\mu \mathrm{m}^{2}\right)$ & 45,77 & $10,45^{\text {abce }}$ & 26,32 & 0,18 & $0,02^{\text {abce }}$ & 0,24 & & \\
\hline Área total fibras $\left(\mu \mathrm{m}^{2}\right)$ & \multicolumn{2}{|c|}{$393.781,44115.392,95$} & & 275,40 & 74,91 & & & \\
\hline Área relativa fibras (\%) & 58,67 & 5,32 & & 0,04 & 0,01 & & & \\
\hline Densidad fibras $\left(\mathrm{n}^{\circ} / \mathrm{mm}^{2}\right)$ & $13.382,57$ & $3.865,85$ & & $2.326,98$ & 587,81 & & & \\
\hline Diámetro fibras $(\mu \mathrm{m})$ & 7,45 & $0,82^{\text {abce }}$ & 2,24 & 0,44 & 0,03 abce & 0,23 & & \\
\hline Área axón $\left(\mu m^{2}\right)$ & 20,26 & 4,97 abce & 12,45 & & & & & \\
\hline Diámetro axón $(\mu \mathrm{m})$ & 5,00 & $0,59^{\text {abce }}$ & 1,60 & & & & & \\
\hline Grosor mielina $(\mu \mathrm{m})$ & 1,22 & $0,12^{\text {abce }}$ & 0,39 & & & & & \\
\hline $\begin{array}{l}\text { Diam, axón/Diam, fibra } \\
(\mu \mathrm{m})\end{array}$ & 0,67 & $0,01^{\text {abce }}$ & 0,05 & & & & & \\
\hline \multicolumn{9}{|l|}{ GRUPO E (4 años) } \\
\hline $\begin{array}{l}\text { Área media del nervio } \\
\left(\mu \mathrm{m}^{2}\right)\end{array}$ & & & & & & & 1.325 .245 & $357.447,54^{\mathrm{abcd}}$ \\
\hline Número fibras & $10.131,75$ & $1.779,81^{\mathrm{a}}$ & & $1.557,94$ & $371,25^{\mathrm{abcd}}$ & & & \\
\hline Porcentaje fibras & 86,67 & 3,55 & & 13,33 & 3,55 & & & \\
\hline Área fibras $\left(\mu \mathrm{m}^{2}\right)$ & 91,05 & $18,34^{\text {abcd }}$ & 73,76 & 0,22 & $0,22^{\text {abcd }}$ & 0,32 & & \\
\hline Área total fibras $\left(\mu \mathrm{m}^{2}\right)$ & \multicolumn{2}{|c|}{$930.916,97303.278,04$} & & 287,39 & 212,34 & & & \\
\hline Área relativa fibras (\%) & 69,91 & 6,80 & & 0,02 & 0,02 & & & \\
\hline Densidad fibras $\left(\mathrm{n}^{\circ} / \mathrm{mm}^{2}\right)$ & $7.853,16$ & $1.302,02$ & & $1.068,58$ & 199,46 & & & \\
\hline Diámetro fibras $(\mu \mathrm{m})$ & 10,08 & $0,91^{\text {abcd }}$ & 4,25 & 0,46 & $0,25^{\mathrm{abcd}}$ & 0,27 & & \\
\hline Área axón $\left(\mu m^{2}\right)$ & 36,64 & $5,51^{\text {abcd }}$ & 29,91 & & & & & \\
\hline Diámetro axón $(\mu \mathrm{m})$ & 6,48 & $0,47^{\text {abcd }}$ & 2,68 & & & & & \\
\hline Grosor mielina $(\mu \mathrm{m})$ & 1,80 & $0,28^{\text {abcd }}$ & 0,90 & & & & & \\
\hline $\begin{array}{l}\text { Diam, axón/Diam, fibra } \\
(\mu \mathrm{m})\end{array}$ & 0,65 & $0,02^{\text {abcd }}$ & 0,06 & & & & & \\
\hline
\end{tabular}

M, fibras mielínicas; AM, fibras amielínicas; Std inter, desviación estándar de la media; std intra, media de la desviación estándar de cada grupo, Los

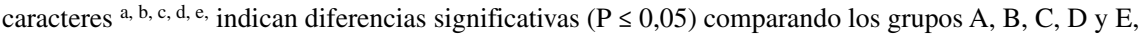

En el cuadro 1 se muestran los valores medios y la variabilidad (media std intra e inter std) para las variables en las fibras mielínicas y amielínicas.

\section{DISCUSIÓN}

En el perro los estudios realizados sobre las características morfométricas de las fibras en los nervios oculomotores son poco abundantes. Sin embargo, estos nervios en otras especies de mamíferos han sido ampliamente estudiados. Sharma y col (2009) estudiaron el desarrollo postnatal del nervio oculomotor en el hombre, y evaluaron el área de los nervios, el área del axón, y el grosor de la vaina de mielina de las fibras mielínicas. Fraher $\left(1989^{a}\right)$ analizó la composición de las fibras de los nervios oculomotor, troclear y abducente en ratas adultas. Ellos mostraron que las fibras mielínicas son más abundantes en el nervio troclear y las fibras amielínicas en el nervio abducente. La composición de los segmentos intracraneales de los nervios oculomotor, troclear y abducente en ovejas fue analizada por Berardinelli y col (2000) que calcularon su tamaño, el número y porcentaje de fibras amielínicas y mielínicas. El nervio oculomotor del perro fue estudiado por Bortolami y col (1977) centrándose especialmente en las fibras amielínicas.

Vivo y col (2013) estudiaron en perros cómo se modifican con el crecimiento algunas características morfométricas de las fibras amielínicas y mielínicas del nervio troclear. Las mismas variables habían sido previamente descritas para el nervio troclear en perros adultos por Vivo y col (2004) y para los tres nervios oculomotores por Vivo y col (2006).

En el presente estudio las fibras amielínicas se observaron en todos los nervios analizados, con diferentes tamaños y distribuidas entre las fibras mielínicas. El número de fibras mielínicas varió de 6.702,67 en los perros más jóvenes a 10.131,75 en los más viejos (cuadro 1). Se supone que la mayor parte de las fibras amielínicas serán mielinizadas durante el crecimiento, como habían indicado previamente Vivo y col (2013). Por su parte, el número de fibras amielínicas disminuyó con la edad, de 1.894,79 (grupo A) a 1.557,94 (grupo E). En las fibras mielínicas los valores del área relativa de las fibras se incrementaron con la edad; por el contrario, en las fibras amielínicas se apreciaron en mayor proporción en el grupo A, probablemente debido a su mayor número en comparación con 
los otros grupos. Respecto de la densidad de la fibra, el desarrollo causó una disminución de sus valores, siendo para las fibras mielínicas $23.103,65 \mu \mathrm{m}^{2}$ en el grupo A y $7.853,16 \mu \mathrm{m}^{2}$ en el grupo E, y para las amielínicas, $6.191,92 \mu \mathrm{m}^{2}$ en el grupo A y $1.068,58 \mu \mathrm{m}^{2}$ en el grupo E. Son el número de fibras y el área del nervio los que determinan la densidad de las fibras. Entre otras variables morfométricas, la densidad de las fibras y sus cambios con el crecimiento ha sido ampliamente utilizada para describir un nervio normal y aquellos afectados por diferentes tipos de patologías nerviosas. Mediante el análisis de nervio sural en ratas Jeronimo y col (2005) indicaron que tanto la disminución en el número de fibras en los trastornos neurológicos como el crecimiento del tejido conectivo del endoneuro alteraban de manera significativa la densidad de las fibras. Como se muestra en los histogramas de los resultados de este estudio (figura 3) los diámetros de las fibras mielínicas variaron con la edad en sus valores máximos, siendo 9,07 para el grupo A, y 21,98 para el grupo E. En consecuencia, hubo una disminución en el porcentaje de las fibras de cada intervalo. En las fibras amielínicas la mayoría de las fibras en los cinco grupos analizados estaban distribuidas entre los intervalos de 0,1 a 1,3 , aunque con el crecimiento se colocaron el mayor número de fibras en los intervalos más bajos (figura 6). En consecuencia, se puede suponer que las fibras más grandes se sometieron a un proceso de mielinización, particularmente entre los grupos B y C (figura 5).

En las fibras mielínicas todas las variables en los grupos A, B, C y D mostraron gráficamente una presentación unimodal. Los perros adultos (grupo E) tenían una disposición bimodal solo para el grosor de la vaina de mielina y el diámetro fibrilar. Como el tamaño del axón tenía una distribución unimodal, la disposición bimodal se la confiere el grosor de la vaina de mielina. En las fibras amielínicas todos los grupos mostraron una presentación unimodal para el área y el diámetro fibrilar (figura 6).

Los resultados de Vivo y col (2006) de las características morfométricas del nervio oculomotor en perros adultos Pastores Alemanes están de acuerdo, en general, con la de los perros adultos Beagle (grupo E) del presente estudio, con la excepción de que el número de fibras mielínicas eran $15 \%$ mayor y el número y área de las fibras amielínicas eran $10 \%$ mayor en perros Beagle.

El rango del diámetro de las fibras mielínicas para el nervio oculomotor en perros (grupo E) de la presente investigación fue de mayor dimensión que el de la rama del nervio oculomotor para el músculo oblicuo inferior encontrado en monos por Ruskell (1983), ligeramente superior al señalado en el gato por Batini y col (1979) y análogo a los aportados en ovejas por Berardinelli y col (2000). La presentación bimodal encontrada para el diámetro fibrilar en perros Beagle del presente estudio ha sido señalada previamente en ovejas por Berardinelli y col (2000). Sin embargo, los valores que separan ambas poblaciones fueron algo mayores en los perros. Como
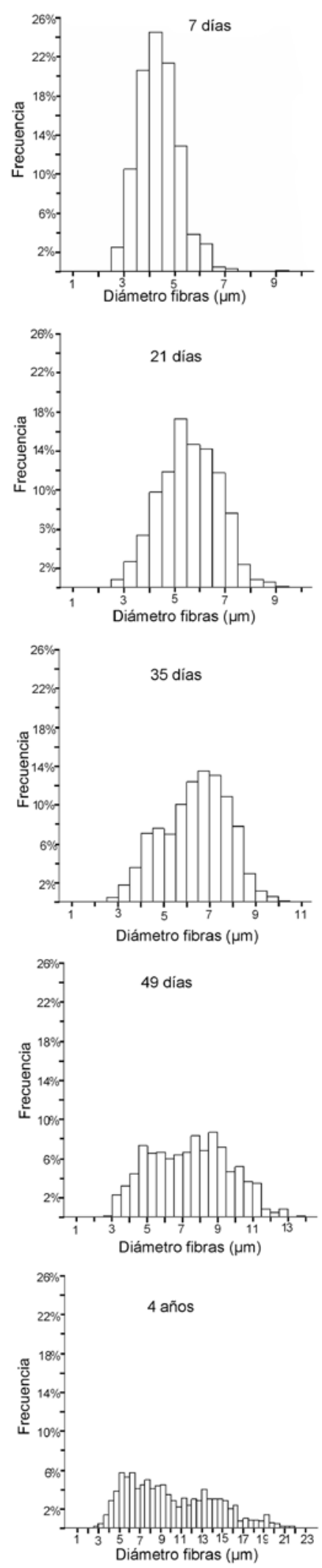

Figura 3. Histogramas de los diámetros de las fibras mielínicas del nervio oculomotor en los cinco grupos de edad estudiados.

Histograms of myelinated fibre diameters of the oculomotor nerve in the five age groups studied. 


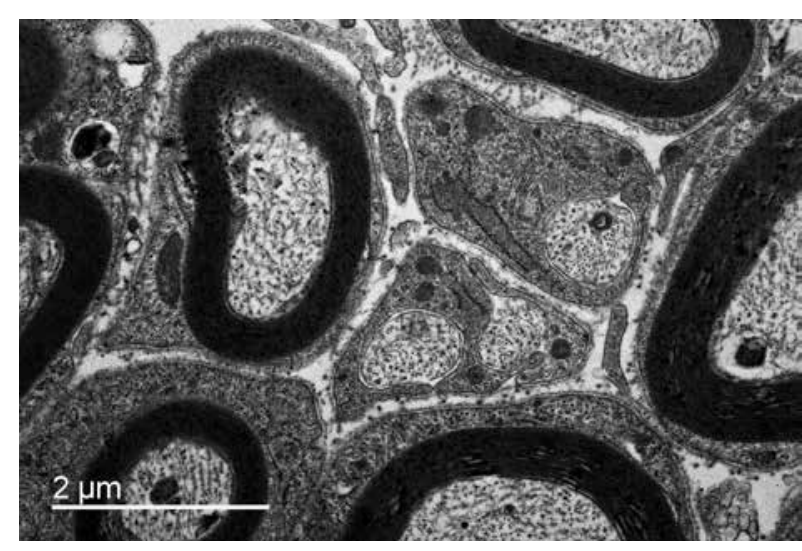

Figura 4. Micrografía electrónica (25.000x) del nervio oculomotor en el grupo A. Se pueden observar fibras mielínicas y amielínicas.

Electron micrograph $(25,000 x)$ of the oculomotor nerve in group A. Myelinated and unmyelinated fibres can be observed.

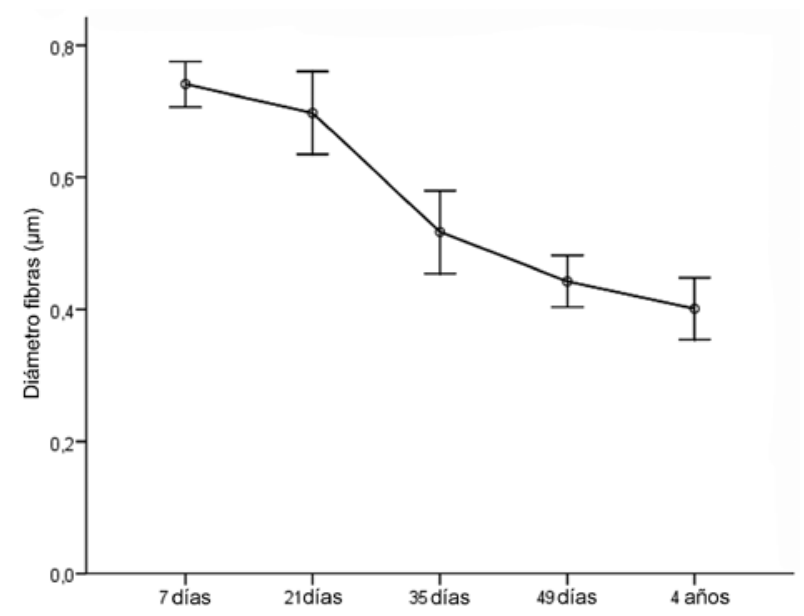

Figura 5. Evolución con la edad del diámetro de las fibras amielínicas.

Evolution with age of the diameter of unmyelinated fibres.

se comentó anteriormente, era el grosor de la vaina de mielina el responsable de esa distribución bimodal para esta variable. La razón entre el diámetro del axón y el diámetro fibrilar del presente estudio determinó valores algo menores que en las ratas analizadas por Fahrenkamp y Friede (1987); Fraher (1989 b y que en ratas y el hombre estudiados por Bardosi y col (1990). Todo lo anterior indica que el grosor de la vaina de mielina es mayor, en estas porciones nerviosas, en perros que en el hombre y ratas.

Los perros del grupo E del presente estudio presentaron un número de fibras mielínicas menor a los señalados en ovejas (Berardinelli y col 2000), y mayor que en ratas (Fraher 1989a) y gatos (Batini y col 1979). El número de fibras amielínicas era menor que en ovejas (Berardinelli y col 2000), y mayor que en ratas (Fraher 1989a). Los resultados de Abo-El-Enene (1978) mostraron que, tanto en el hombre como en ovejas, un número importante de
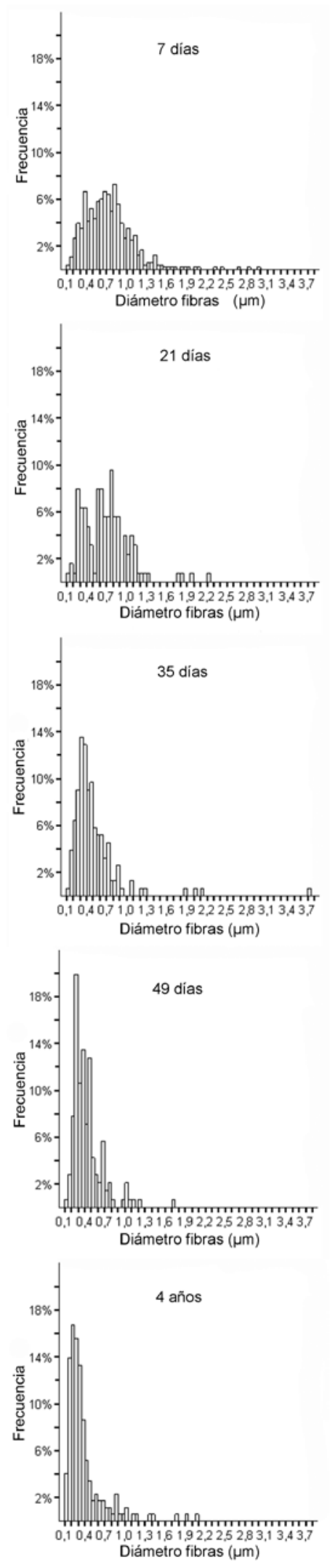

Figura 6. Histogramas de los diámetros de las fibras amielínicas del nervio oculomotor en los cinco grupos de edad estudiados.

Histograms of unmyelinated fibre diameters of the oculomotor nerve in the five age groups studied. 
las fibras nerviosas mielínicas del nervio oculomotor eran fibras propioceptivas grandes y pequeñas. Se puede suponer que en el mismo nervio del perro no todas las fibras mielínicas son neuronas motoras y que un número considerable de ellas, al igual que en otras especies de mamíferos, tienen función propioceptiva.

Comparando entre los mismos grupos de edad, el número de fibras nerviosas mielínicas y amielínicas del nervio oculomotor del presente estudio fue considerablemente mayor que los aportados por Vivo y col (2013) para el nervio troclear. Esto era lo esperado, ya que el número de fibras de un nervio en particular depende del número de músculos inervados por este nervio, en este caso cinco el nervio oculomotor y uno el nervio troclear.

En este trabajo, el área del nervio presentó valores mayores que los indicados por Vivo y col (2013) para el nervio troclear. En las fibras mielínicas el área de todas las fibras fue mucho mayor y el diámetro de las fibras y el área de las fibras ligeramente inferior a los indicados por Vivo y col (2013). Sin embargo, en este estudio, como el diámetro de la fibra fue menor y el diámetro del axón fue mayor, el grosor de la vaina de mielina fue algo menor. En las fibras amielínicas el área de todas las fibras, el área de las fibras y el diámetro fibrilar eran mucho mayores que en el estudio de Vivo y col (2013) sobre el nervio troclear.

En resumen, en los perros desde la edad de una semana (grupo A) a la edad adulta (grupo E) el área de la sección transversal del nervio oculomotor aumentó de tamaño 4,5 veces; el número de fibras mielínicas aumentó significativamente 1,5 veces y el diámetro de estas fibras 2,3 veces; mientras tanto, el número de fibras amielínicas disminuyó en $20 \%$ y su diámetro $40 \%$ desde el grupo A al grupo E.

Los resultados del presente estudio contribuyen al conocimiento de los valores normales de algunas características morfométricas de las fibras mielínicas y amielínicas del nervio oculomotor en perros Beagle durante el crecimiento, y pueden servir como una referencia ante aquellos animales que presenten patologías nerviosas que cursen con alteraciones de estas características.

\section{REFERENCIAS}

Abo-El-Enene MA. 1978. Propioceptive afferent fibers in the cranial nerves III, IV and VI. Acta Anat 101, 62-65.

Bardosi A, J Sallo, C Schafer, H Muhlendy. 1990. Morphometric comparison between human and rat abducens and oculomotor nerves. Acta Anat 138, 24-31.

Batíni C, C Buisseret-Delmas, RT Kado. 1979. On the fibers of the III, IV and VI cranial nerves of the cat. Arch Ital Biol 117, 111-122.

Berardinelli P, PA Scapolo, AM Barazzoni, V Russo, R Bortolami. 2000. Nerve fiber composition of the intracranial portion of the oculomotor, trochlear, and abducens nerves in the sheep. Anat Rec 260, 294-298.

Bortolami R, A Veggetti, E Callegari, ML Lucchi, G Palmieri. 1977. Afferent fibers and sensory ganglion cells within the oculomotor nerve in some mammals and man. I. Anatomical investigations. Arch Ital Biol 115, 355-386.

Brodal A. 1981. Neurological anatomy in relation to clinical medicine. Oxford University Press, Oxford, UK.

Fahrenkamp I, RL Friede. 1987. Characteristic variations of relative myelin sheath thickness in 11 nerves of the rat. Anat Embryol 177, 115-121.

Fraher JP. 1989 . Axon numbers in rat oculomotor, trochlear and abducent nerves. J Anat 166, 151-155.

Fraher JP. 1989 $9^{\mathrm{b}}$. Axon-myelin relationships in rat cranial nerves III, IV, and VI: a morphometric study of large and small-fiber classes. J Comp Neurol 286, 384-390.

Hayakawa T, M Itoh, T Miki, T Kaneto, H Tomiyama, Y Takeuchi. 2000. Sympathetic fiber innervating the extraocular muscles: cells of origin in the cat superior cervical ganglion. Okajimas Folia Anat Jpn 77, 119-124.

Jeronimo A, CDA Jeronimo, OA Rodrigues Fiho, LS Sanada, VPS Fazan. 2005. Microscopic anatomy of the sural nerve in the postnatal developing rat: a longitudinal and lateral symmetry study. J Anat 206, 93-100.

Kawamura N, K Matsumoto, A Wada, N Goto. 2000. Unmyelinated nerve fiber analysis of the human oculomotor nerve. Okajimas Folia Anat Jpn 77, 59-62.

König HE, HG Liebich. 2014. Veterinay anatomy of domestic mammals. Textbook and colour atlas. Schattauer $\mathrm{GmbH}$, Stuttgart, Germany.

Lanzino G, A Andreoli, F Tognetti, P Limoni, F Calbucci, R Bortolami, ML Lucchi, E Callegari, C Testa. 1993. Orbital pain and unruptured carotid-posterior communicating artery aneurysms: the role of sensory fibers of the third cranial nerve. Acta Neurochir (Wien) 120, 7-11.

Manni E, R Bortolami, VE Pettorossi, ML Lucchi, E Callegari. 1978. Afferent fibers and sensory ganglion cells within the oculomotor nerve in some mammals and man. II. Electrophysiological investigations. Arch Ital Biol 116, 16-24.

Manni E, R Bortolami, VE Pettorossi, ML Lucchi, E Callegari, F Draicchio. 1987. Influence of oculomotor nerve afferents on central endings of primary trigeminal fibers. Arch Ital Biol 126, 29-39.

Manni E, F Draicchio, VE Pettorossi, C Carobi, S Grassi, R Bortolami, ML Lucchi. 1989. On the nature of the afferent fibers of oculomotor nerve. Arch Ital Biol 127, 99-108.

Mayhew TM, AK Sharma. 1984a . Sampling schemes for estimating nerve size. I. Methods for nerve trunks of mixed fascicularity. $J$ Anat 139, 45-58.

Mayhew TM, AK Sharma. 1984 . Sampling schemes for estimating nerve size. II. Methods for unifascicular nerve trunks. J Anat 139, 59-66.

Porter JD, RF Spencer. 1982. Localization of morphology of cat extraocular muscle afferent neurones identified by retrograde transport of horseradish peroxidase. J Comp Neurol 204, 56-64.

Ruskell GL. 1983. Fibre analysis of the nerve to the inferior oblique muscle in monkeys. J Anat 137, 445-455.

Sharma S, B Ray, D Bhardwaj, AK Dwivedi, TS Roy. 2009. Age changes in the human oculomotor nerve. A stereological study. Ann Anat 191, 260-266.

Vivo J, JL Morales, A Díz, AM Galisteo, JG Monterde, A Blanco, E Agüera. 2004. Intracranial portion of the nerve and dorsal oblique muscle composition in dog: a structural and ultrastructural study. J Morphol 262, 708-713.

Vivo J, JL Morales, A Díz, AM Galisteo, JG Monterde, E Agüera, F Miró. 2006. Structural and ultrastructural study of the intracranial portion of the oculomotor, trochlear and abducent nerves in dog. Anat Histol Embriol 35, 184-189.

Vivo J, AM Galisteo, F Miró, E Agüera, C Plaza, A Díz. 2013. Morphometric changes in the trochlear nerve with growth. Anat Histol Embriol 42, 183-190. 